\title{
List of assessors
}

We would like to thank the following who have acted as specialist assessors during the past year.

D. Abrahamson
C.E. Adams
G. Andrews
B. Ballinger
T.R.E. Barnes
K. Bergmann
M. Bernadt
M. Berelowitz
G.E. Berrios
S. Bhate
D. Bhugra
D. Black
N. Bouras
P.M.A. Bowden
W.D. Boyd
P. Bruggen
W.F. Bynum
F. Caldicott
H.R. Cattell
J. Chick
D. Chiswick
J. Coid
M. Collins
L. Cooke
J. Cookson
A. Cotgrove
D. Cottrell
J.L. Cox
J.L. Crammer
A. Crisp
L. Culliford
J. Cutting
J. Dalton
J. Dare
A. David
N. Davies
K. Davison
K.A. Day
C. Dean
J. Deyoy
L. Drummond
R. Duffett

J.A.T. Dyer
K. Ebmeir
L.H. Fagin
A. Farmer
R. Farmer
P.Fenwick
E. Finch
N. Fisher
J. Fisk
M. Fitzgerald
D.G. Fowlie
W. Fraser
P. Freeling
C.P.L. Freeman
R. Fry
M.E. Garralda
R. Gater
A. Gath
A. Ghodse
I. Glass
M. Greenberg
J. Greenwood
A.T. Grounds
D. Grubin
A. Hall
M.A. Harper
F. Hassanyeh
K. Hawton
D. Healy
J. Herzberg
A. Higgitt
J. Higgins
P. Hill
P. Hoare
S. Hirsch
N.L. Holden
F. Holloway
J. Holmes
M. Humphreys
L. Ions
A. Jacques
A.J. Jaffa

D. Johnson

D.J. Jolley

J.R. Jones

P. Joseph

O. Junaid

C.A. Kaplan

C.L.E. Katona

R.F. Kehoe

A.M.P. Kellam

H. Kennedy

S.R. Kisley

G.E. Langley

G. Lewis

S. Lewis

R. Levy

M.H. Lim

C.S. Littlejohns

R. Littlewood

K. Lloyd

R.M. Lyall

A. McFadyen

A.A. McKechnie

C. McWilliam

C. Mace

K. Malcolm

F.R. Margison

G. Masterton

J. Mathal

D. Mawson

S. Milne

S. Montgomery

M. Murphy

J. O'Hara

R.L. Palmer

C.M. Parkes

S. Payne

A.J. Pelosi

J.S. Pippard

M. Potter

S. Potts

G. Pullen

J.H. Race

F. Reader

A.H. Reid

B. Ritson

S. Ritter

J. Roberts

J.R. Robertson

T.D. Rogers

O. Russell

L. Scarth

D. Scott

J.L. Scott

C.P. Seager

M.A. Serfaty

M.F. Shanks

P. Shoenberg

M. Silverman

J. Smith

D. Somekh

J. Spencer

J. Strachan

G. Stein

R.S. Stern

M. Tannahill

D. Tantam

P.J. Taylor

D. Thompson

G. Thornicroft

S.P. Tyrer

C. Van Velsen

G.E.T. Vincenti

J.P. Watson

J.P. Wattis

P. Whewell

J.A. Whitehead

S. Whyte

S.R. Wilson

J. Wing

R. Wrate

A.J. Yellowlees

H. Zeitlin

G. Pullen

